\title{
Kantowski-Sachs String Cosmological Model with Bulk Viscosity in General Scalar Tensor Theory of Gravitation
}

\author{
V. U. M. Rao and D. Neelima \\ Department of Applied Mathematics, Andhra University, Visakhapatnam, Andhra Pradish 530003, India \\ Correspondence should be addressed to V. U. M. Rao; umrao57@hotmail.com
}

Received 8 April 2013; Accepted 8 May 2013

Academic Editors: N. E. Bjerrum-Bohr, P. Hogan, and M. Znojil

Copyright ( 2013 V. U. M. Rao and D. Neelima. This is an open access article distributed under the Creative Commons Attribution License, which permits unrestricted use, distribution, and reproduction in any medium, provided the original work is properly cited.

\begin{abstract}
A new class of spatially homogeneous Kantowski-Sachs string cosmological models with bulk viscosity in Nordtvedt (1970) general scalar-tensor theory of gravitation with the help of a special case proposed by Schwinger (1970) is obtained. In this paper we have presented anisotropic as well as isotropic cosmological models. Some important features of the models, thus obtained, have been discussed. These exact models are new and more general and represent not only the early stages of evolution but also the present universe.
\end{abstract}

\section{Introduction}

Nordtvedt [1] proposed a general class of scalar-tensor gravitational theories in which the parameter $\omega$ of the BD theory is allowed to be an arbitrary (positive definite) function of the scalar field $[\omega \rightarrow \omega(\phi)]$. This general class of scalar-tensor gravitational theories includes the Jordan [2] and BransDicke [3] theories as special cases.

Barker [4], Ruban and Finkelstein [5], Banerjee and Santos [6,7], and Shanti and Rao $[8,9]$ are some of the authors who have investigated several aspects of Nordtvedt general scalar-tensor theory. Rao and Kumari [10] have discussed a cosmological model with negative constant deceleration parameter in this theory, and very recently Rao et al. [11] have obtained Kaluza-Klein radiating model in a general scalartensor theory of gravitation.

The field equations of general scalar-tensor theory proposed by Nordtvedt are

$$
\begin{aligned}
R_{i j}-\frac{1}{2} g_{i j} R= & -8 \pi \phi^{-1} T_{i j}-\omega \phi^{-2}\left(\phi_{,_{i}} \phi_{,_{j}}-\frac{1}{2} g_{i j} \phi_{,_{k}} \phi^{, k}\right) \\
& -\phi^{-1}\left(\phi_{i ; j}-g_{i j} \phi_{; k}^{, k}\right), \\
\phi_{j k}^{, k} & =\frac{8 \pi T}{3+2 \omega}-\frac{1}{(3+2 \omega)} \frac{d \omega}{d \phi} \phi_{,_{i}} \phi^{i},
\end{aligned}
$$

where $R_{i j}$ is the Ricci tensor, $R$ is the scalar curvature, $T_{i j}$ is the stress energy tensor of the matter, and comma and semicolon denote partial and covariant differentiation, respectively.

Also, we have

$$
T_{; j}^{i j}=0
$$

which is a consequence of the field equations (1).

Beside the Bianchi type metrics, the Kantowski-Sachs [12] models are also describing spatially homogeneous universes. For a review of Kantowski-Sachs of metrics one can refer to MacCallum [13]. These metrics represent homogeneous but anisotropically expanding (or contacting) cosmologies and provide models where the effects of anisotropic can be estimated and compared with all well-known FriedmannRobertson-Walker class of cosmologies. Wang [14] has obtained Kantowski-Sachs string cosmological model with bulk viscosity in general relativity. Kandalkar et al. [15] have discussed Kantowski-Sachs viscous fluid cosmological model with a varying $\Lambda$. Kandalkar et al. [16] have obtained string cosmology in Kantowski-Sachs space-time with bulk viscosity and magnetic field. Rao et al. [17] have studied various Bianchi type string cosmological models in the presence of bulk viscosity. Recently Rao et al. [18] and Rao and Sireesha [19] have obtained Bianchi types II, VIII, and IX string cosmological models with bulk viscosity in Lyra [20] and Brans and Dicke [3] theory of gravitation, respectively. 
Motivated by the above investigations, we study spatially homogeneous and anisotropic Kantowski-Sachs string cosmological model with bulk viscosity in Nordtvedt [1] general scalar-tensor theory with the help of a special case proposed by Schwinger [21], that is, $3+2 \omega(\phi)=1 / \lambda \phi$, where $\lambda$ is a constant.

\section{Metric and Energy Momentum Tensor}

We consider a spatially homogeneous Kantowski-Sachs metric of the form

$$
d s^{2}=d t^{2}-A^{2} d r^{2}-B^{2}\left(d \theta^{2}+\sin ^{2} \theta d \phi^{2}\right),
$$

where $A$ and $B$ are the functions of time $t$ only.

The energy momentum tensor for a bulk viscous fluid containing one-dimensional string is

$$
\begin{gathered}
T_{i j}=(\rho+\bar{p}) u_{i} u_{j}-\bar{p} g_{i j}-\lambda_{s} x_{i} x_{j}, \\
\bar{p}=p-3 \xi H,
\end{gathered}
$$

where $p=\epsilon_{0} \rho\left(0 \leq \epsilon_{0} \leq 1\right)$. Here $\bar{p}$ is the total pressure which includes the proper pressure $p, \rho$ is the rest energy density of the system, $\lambda_{s}$ is the tension in the string, $\xi(t)$ is the coefficient of bulk viscosity, $3 \xi H$ is usually known as bulk viscous pressure, $H$ is the Hubble parameter, $u^{i}$ is the four velocity vector, and $x^{i}$ is a space-like vector which represents the anisotropic directions of the string.

Here $u^{i}$ and $x^{i}$ satisfy the equations

$$
\begin{gathered}
g_{i j} u^{i} u^{j}=1, \\
g_{i j} x^{i} x^{j}=-1, \\
u^{i} x_{i}=0 .
\end{gathered}
$$

We assume that the string is to be lying along the $x$-axis. The one-dimensional strings are assumed to be loaded with particles, and the particle energy density is

$$
\rho_{p}=\rho-\lambda_{s}
$$

In a comoving coordinate system, we get

$$
T_{1}^{1}=\lambda_{s}-\bar{p}, \quad T_{2}^{2}=T_{3}^{3}=-\bar{p}, \quad T_{4}^{4}=\rho,
$$

where $\rho, \lambda_{s}, \bar{p}$, and $\phi$ are functions of time $t$ only.

\section{Solution of the Field Equations}

Using comoving coordinates, the field equations (1) to (2) for the metric (3) with the help of (4) to (8) can be written as

$$
\begin{aligned}
& 2 \frac{\ddot{B}}{B}+\frac{\dot{B}^{2}}{B^{2}}+\frac{1}{B^{2}}+\frac{\omega \dot{\phi}^{2}}{2 \phi^{2}}+\frac{\ddot{\phi}}{\phi}+2 \frac{\dot{\phi} \dot{B}}{\phi B}=\frac{8 \pi\left(\lambda_{s}-\bar{p}\right)}{\phi}, \\
& \frac{\ddot{A}}{A}+\frac{\ddot{B}}{B}+\frac{\dot{A} \dot{B}}{A B}+\frac{\omega \dot{\phi}^{2}}{2 \phi^{2}}+\frac{\ddot{\phi}}{\phi}+\frac{\dot{\phi}}{\phi}\left(\frac{\dot{A}}{A}+\frac{\dot{B}}{B}\right)=-\frac{8 \pi \bar{p}}{\phi},
\end{aligned}
$$

$$
\begin{gathered}
2 \frac{\dot{A} \dot{B}}{A B}+\frac{\dot{B}^{2}}{B^{2}}+\frac{1}{B^{2}}-\frac{\omega \dot{\phi}^{2}}{2 \phi^{2}}+\frac{\dot{\phi}}{\phi}\left(\frac{\dot{A}}{A}+2 \frac{\dot{B}}{B}\right)=\frac{8 \pi \rho}{\phi}, \\
\ddot{\phi}+\dot{\phi}\left(\frac{\dot{A}}{A}+2 \frac{\dot{B}}{B}\right)=\frac{8 \pi}{(3+2 \omega)}\left(\rho+\lambda_{s}-3 \bar{p}\right) \\
-\frac{1}{(3+2 \omega)} \frac{d \omega}{d \phi} \dot{\phi}^{2}, \\
\dot{\rho}+(\rho+\bar{p})\left(\frac{\dot{A}}{A}+2 \frac{\dot{B}}{B}\right)-\lambda_{s} \frac{\dot{A}}{A}=0 .
\end{gathered}
$$

Here the overhead dot denotes differentiation with respect to $t$.

By using the transformation $d t=A B^{2} d T$, the above field equations (9) can be written as

$$
\begin{aligned}
& 2 \frac{B^{\prime \prime}}{B}- 3 \frac{B^{\prime 2}}{B^{2}}-2 \frac{A^{\prime} B^{\prime}}{A B}+A^{2} B^{2}+\frac{\omega}{2} \frac{\phi^{\prime 2}}{\phi^{2}}+\frac{\phi^{\prime \prime}}{\phi}-\frac{\phi^{\prime}}{\phi} \frac{A^{\prime}}{A} \\
&=\frac{8 \pi}{\phi}\left(\lambda_{s}-\bar{p}\right)\left(A^{2} B^{4}\right) \\
& \frac{A^{\prime \prime}}{A}- \frac{A^{\prime 2}}{A^{2}}+\frac{B^{\prime \prime}}{B}-2 \frac{B^{\prime 2}}{B^{2}}-2 \frac{A^{\prime} B^{\prime}}{A B}+\frac{\omega}{2} \frac{\phi^{\prime 2}}{\phi^{2}}+\frac{\phi^{\prime \prime}}{\phi}-\frac{\phi^{\prime}}{\phi} \frac{B^{\prime}}{B} \\
&=\frac{-8 \pi \bar{p}}{\phi}\left(A^{2} B^{4}\right), \\
& 2 \frac{A^{\prime} B^{\prime}}{A B}+\frac{B^{\prime 2}}{B^{2}}+A^{2} B^{2}-\frac{\omega}{2} \frac{\phi^{\prime 2}}{\phi^{2}}+\frac{\phi^{\prime}}{\phi}\left(\frac{A^{\prime}}{A}+2 \frac{B^{\prime}}{B}\right) \\
&=\frac{8 \pi}{\phi} \rho\left(A^{2} B^{4}\right), \\
& \rho^{\prime}+(\rho+\bar{p})\left(\frac{A^{\prime}}{A}+2 \frac{B^{\prime}}{B}\right)-\lambda_{s}\left(\frac{A^{\prime}}{A}\right)=0 \\
&(3+2 \omega) \phi^{\prime \prime}=8 \pi\left(\rho-3 \bar{p}+\lambda_{s}\right)\left(A^{2} B^{4}\right)-\phi^{\prime 2} \frac{d \omega}{d \phi}
\end{aligned}
$$

From hereafter the overhead dash denotes differentiation with respect to $T$.

The field equations (10) to (13) are four independent equations with seven unknowns $A, B, \omega, \phi, \rho, \bar{p}$, and $\lambda_{s}$.

From (10) to (13), we have

$$
\begin{aligned}
(3+ & 2 \omega) \phi^{\prime \prime}+\frac{d \omega}{d \phi} \phi^{\prime 2} \\
= & 2 \phi\left[\frac{A^{\prime \prime}}{A}-\frac{A^{\prime 2}}{A^{2}}+2 \frac{B^{\prime \prime}}{B}-3 \frac{B^{\prime 2}}{B^{2}}-2 \frac{A^{\prime} B^{\prime}}{A B}+A^{2} B^{2}\right] \\
& +\omega \frac{\phi^{\prime 2}}{\phi}+3 \phi^{\prime \prime} .
\end{aligned}
$$


Here we obtain string cosmological model with bulk viscosity in Nordtvedt's general scalar-tensor theory with the help of a special case proposed by Schwinger [21] in the form

$$
3+2 \omega(\phi)=\frac{1}{\lambda \phi}, \quad \lambda=\text { constant }
$$

From (15) and (16), we get

$$
\begin{aligned}
& \frac{1}{\lambda}\left[\frac{\phi^{\prime \prime}}{\phi}-\frac{\phi^{\prime 2}}{\phi^{2}}\right]+\frac{3}{2} \frac{\phi^{\prime 2}}{\phi}-3 \phi^{\prime \prime} \\
& \quad=2 \phi\left[\frac{A^{\prime \prime}}{A}-\frac{A^{\prime 2}}{A^{2}}+2 \frac{B^{\prime \prime}}{B}-3 \frac{B^{\prime 2}}{B^{2}}-2 \frac{A^{\prime} B^{\prime}}{A B}+A^{2} B^{2}\right]
\end{aligned}
$$

In order to solve the above equation completely, we assume that the expansion scalar is proportional to shear scalar. This condition leads to

$$
A=B^{n}, \quad n \neq 0 .
$$

From (17) and (18), we get

$$
\phi=e^{\left(k_{1} T+k_{2}\right)},
$$

where $k_{1}$ and $k_{2}$ are arbitrary constants and

$$
\frac{B^{\prime \prime}}{B}-\frac{3(n+1)}{n+2} \frac{B^{\prime 2}}{B^{2}}=\frac{-1}{(n+2)} B^{2 n+2}-\frac{3}{4(n+2)} k_{1}^{2} .
$$

From (20), we get

$$
B=\left[\left(\frac{k_{4}}{k_{3}}\right) \operatorname{sech}(n+1) k_{4} T\right]^{1 /(n+1)}, \quad n \neq-1,
$$

where $k_{3}^{2}=1 /\left(n^{2}+n+1\right)$ and $k_{4}^{2}=3 k_{1}^{2} / 4(2 n+1)$.

From (18) and (21), we get

$$
A=\left[\left(\frac{k_{4}}{k_{3}}\right) \operatorname{sech}(n+1) k_{4} T\right]^{n /(n+1)} .
$$

From (12), we get the string energy density

$$
\begin{gathered}
\rho=\frac{1}{8 \pi k_{5}}\left[\cosh (n+1) k_{4} T\right]^{2(n+2) /(n+1)} \\
\times\left\{e ^ { ( k _ { 1 } T + k _ { 2 } ) } \left[(2 n+1) k_{4}^{2} \tanh ^{2}(n+1) k_{4} T\right.\right. \\
+\frac{k_{4}^{2}}{k_{3}^{2}} \operatorname{sech}^{2}(n+1) k_{4} T \\
\left.-(n+2) k_{1} k_{4} \tanh (n+1) k_{4} T+\frac{3}{4} k_{1}^{2}\right] \\
\left.-\frac{k_{1}^{2}}{4 \lambda}\right\},
\end{gathered}
$$

where $k_{5}=\left(k_{4} / k_{3}\right)^{2(n+2) /(n+1)}$.
From (11), we get the total pressure

$$
\begin{gathered}
\bar{p}=\frac{1}{8 \pi k_{5}}\left[\cosh (n+1) k_{4} T\right]^{2(n+2) /(n+1)} \\
\times\left\{e ^ { ( k _ { 1 } T + k _ { 2 } ) } \left[(2 n+1) k_{4}^{2} \tanh ^{2}(n+1) k_{4} T\right.\right. \\
+(n+1)^{2} k_{4}^{2} \operatorname{sech}^{2}(n+1) k_{4} T \\
\left.-k_{1} k_{4} \tanh (n+1) k_{4} T-\frac{1}{4} k_{1}^{2}\right] \\
\left.-\frac{k_{1}^{2}}{4 \lambda}\right\} .
\end{gathered}
$$

The proper pressure is given by

$$
\begin{gathered}
p=\frac{\varepsilon_{0}}{8 \pi k_{5}}\left[\cosh (n+1) k_{4} T\right]^{2(n+2) /(n+1)} \\
\times\left\{e ^ { ( k _ { 1 } T + k _ { 2 } ) } \left[(2 n+1) k_{4}^{2} \tanh ^{2}(n+1) k_{4} T\right.\right. \\
+\frac{k_{4}^{2}}{k_{3}^{2}} \operatorname{sech}^{2}(n+1) k_{4} T \\
\left.-(n+2) k_{1} k_{4} \tanh (n+1) k_{4} T+\frac{3}{4} k_{1}^{2}\right] \\
\left.-\frac{k_{1}^{2}}{4 \lambda}\right\} .
\end{gathered}
$$

The coefficient of bulk viscosity is given by

$$
\begin{aligned}
\xi=\frac{1}{8 \pi k_{5}(n+2)}[ & \left.\cosh (n+1) k_{4} T\right]^{2(n+2) /(n+1)} \\
\times\left\{e^{\left(k_{1} T+k_{2}\right)}[[\right. & \left.\left(n^{2}+2\right)\left(1-\varepsilon_{0}\right)+n\left(4-3 \varepsilon_{0}\right)\right] \\
& \times k_{4} \tanh (n+1) k_{4} T \\
& -\frac{\left(3 \varepsilon_{0}+1\right)}{4} \frac{k_{1}^{2}}{k_{4}} \operatorname{coth}(n+1) k_{4} T \\
& \left.+\left(\varepsilon_{0}(n+2)-1\right) k_{4}\right] \\
+\frac{\left(\varepsilon_{0}-1\right)}{4 \lambda} & \left.\frac{k_{1}^{2}}{k_{4}} \operatorname{coth}(n+1) k_{4} T\right\} .
\end{aligned}
$$

From (10) and (11), we get the string tension density

$$
\begin{aligned}
\lambda_{s}= & \frac{1}{8 \pi k_{5}}\left[\cosh (n+1) k_{4} T\right]^{2(n+2) /(n+1)} \\
& \times\left\{e ^ { ( k _ { 1 } T + k _ { 2 } ) } \left[n(2 n+1) k_{4}^{2} \operatorname{sech}^{2}(n+1) k_{4} T\right.\right. \\
& \left.\left.+(n-1) k_{1} k_{4} \tanh (n+1) k_{4} T\right]\right\} .
\end{aligned}
$$


The metric (3) can now be written as

$$
\begin{aligned}
d s^{2}= & \frac{1}{k_{5}}\left[\cosh (n+1) k_{4} T\right]^{2(n+2) /(n+1)} d T^{2} \\
& -\left[\left(\frac{k_{4}}{k_{3}}\right) \operatorname{sech}(n+1) k_{4} T\right]^{2 n /(n+1)} d r^{2} \\
& -\left[\left(\frac{k_{4}}{k_{3}}\right) \operatorname{sech}(n+1) k_{4} T\right]^{2 /(n+1)}\left(d \theta^{2}+\sin ^{2} \theta d \phi^{2}\right) .
\end{aligned}
$$

Thus, for $n \neq 1$, the metric (28) together with (19) and (23) to (27) constitutes a Kantowski-Sachs anisotropic string cosmological model with bulk viscosity in Nordtvedt's general scalar-tensor theory of gravitation with a special case proposed by Schwinger.

3.1. Isotropic Cosmological Model. For $n=1$, the metric (28) together with (19) and (23) to (27) constitutes isotropic string cosmological model with bulk viscosity in Nordtvedt's general scalar-tensor theory of gravitation with a special case proposed by Schwinger.

\section{Some Important Features of the Model}

The volume element of the model (28) is given by

$$
V=(-g)^{1 / 2}=\sqrt{k_{5}}\left[\operatorname{sech}(n+1) k_{4} T\right]^{(n+2) /(n+1)} \sin \theta .
$$

The expression for the expansion scalar $\theta$ is given by

$$
\theta=u^{i},_{i}=-(n+2) k_{4} \tanh (n+1) k_{4} T,
$$

and the shear $\sigma$ is given by

$$
\sigma^{2}=\frac{1}{2} \sigma^{i j} \sigma_{i j}=\frac{7}{18}(n+2)^{2} k_{4}^{2} \tanh ^{2}(n+1) k_{4} T .
$$

The deceleration parameter $q$ is given by

$$
\begin{gathered}
q=-3 \theta^{-2}\left(\theta_{, i} u^{i}+\frac{1}{3} \theta^{2}\right) \\
q=-\left(\frac{-12(n+1)}{(n+2) e^{2(n+1) k_{4} T}\left(1-e^{-2(n+1) k_{4} T}\right)^{2}}+1\right) .
\end{gathered}
$$

The Hubble parameter $H$ is given by

$$
H=\frac{-(n+2) k_{4} \tanh (n+1) k_{4} T}{3} .
$$

The tensor of rotation $w_{i j}=u_{i, j}-u_{j, i}$ is identically zero, and hence this universe is nonrotational.

\section{Conclusions}

In this paper we have presented Kantowski-Sachs string cosmological models with bulk viscosity in Nordtvedt [1] general scalar-tensor theory with the help of a special case proposed by Schwinger [21]. The models presented here are free from singularities, and the spatial volume vanishes as $T \rightarrow \infty$. We observe that, as $T$ approaches to infinity, the expansion scalar $\theta$ leads to a constant value. This shows that the universe expands homogeneously. We also observe that the shear scalar $\sigma$ and the Hubble parameter $H$ approach to constant value as $T$ tends to infinity. The energy density, total pressure, string tension density, and coefficient of bulk viscosity diverge with the increase of time. For this model, the deceleration parameter $q$ is negative for large values of $T$. Hence the model represents accelerating universe and conformed the well-known fact that the scalar field and the bulk viscosity will play a vital role in getting an accelerated universe. For $n=1$, the model represents isotropic universe. These anisotropic as well as isotropic exact models are new, more general, and free from singularities. Also the models presented here represent not only the early stages of evolution but also the present universe.

\section{Acknowledgment}

The second author (D. Neelima) is grateful to the Department of Science and Technology (DST), New Delhi, India, for providing INSPIRE fellowship.

\section{References}

[1] K. Nordtvedt, Jr., "Post-Newtonian metric for a general class of scalar-tensor gravitational theories and observational consequences," The Astrophysical Journal, vol. 161, pp. 1059-1067, 1970.

[2] P. Jordan, Schwerkraft und Weltall, Friedch Vieweg and Sohn, Braunschweig, Germany, 1955.

[3] C. Brans and R. H. Dicke, "Mach's principle and a relativistic theory of gravitation," Physical Review, vol. 124, pp. 925-935, 1961.

[4] B. M. Barker, "General scalar-tensor theory of gravity with constant G," The Astrophysical Journal, vol. 219, pp. 5-11, 1978.

[5] V. A. Ruban and A. M. Finkelstein, "Generalization of the TaubKazner cosmological metric in the scalar-tensor gravitation theory," Lettere Al Nuovo Cimento, vol. 5, no. 3, pp. 289-293, 1972.

[6] A. Banerjee and N. O. Santos, "Homogeneous radiation-filled universe in general scalar tensor theory," Journal of Physics A, vol. 14, no. 10, pp. 2829-2835, 1981.

[7] A. Banerjee and N. O. Santos, "Homogeneous cosmological model in general scalar-tensor theory," Physical Review D, vol. 23, no. 10, pp. 2111-2115, 1981.

[8] K. Shanti and V. U. M. Rao, "Conformally flat static space-time in the general scalar-tensor theory of gravitation," Astrophysics and Space Science, vol. 162, no. 1, pp. 163-165, 1989.

[9] K. Shanthi and V. U. M. Rao, "Bianchi type-VI。 cosmological model in the general scalar-tensor theory of gravitation," Astrophysics and Space Science, vol. 172, no. 1, pp. 83-88, 1990.

[10] V. U. M. Rao and G. S. D. Kumari, "A cosmological model with negative constant deceleration parameter in a general scalartensor theory of gravitation," International Journal of Theoretical Physics, vol. 51, no. 1, pp. 311-315, 2012.

[11] V. U. M. Rao, S. G. Kumari, and B. J. M. Rao, "Kaluza-Klein radiating model in a general scalar-tensor theory," Astrophysics and Space Science, vol. 337, no. 2, pp. 795-798, 2012. 
[12] R. Kantowski and R. K. Sachs, "Some spatially homogeneous anisotropic relativistic cosmological models," Journal of Mathematical Physics, vol. 7, no. 3, pp. 443-446, 1966.

[13] E. A. Follett and T. H. Pennington, "Antiviral effect of constituent parts of the rifampicin molecule," Nature, vol. 230, no. 5289, pp. 117-118, 1971.

[14] X.-X. Wang, "Kantowski-Sachs string cosmological model with bulk viscosity in general relativity," Astrophysics and Space Science, vol. 298, no. 3, pp. 433-440, 2005.

[15] S. P. Kandalkar, P. P. Khade, and S. P. Gawande, "KantowskiSachs viscous fluid cosmological model with a varying," Turkish Journal of Physics, vol. 33, pp. 155-165, 2009.

[16] S. P. Kandalkar, A. P. Wasnik, and S. P. Gawande, "String cosmology in Kantowski Sachs space-time with bulk viscosity and magnetic field," Journal of Vectorial Relativity, vol. 2, pp. 5764, 2011.

[17] V. U. M. Rao, G. S. D. Kumari, and K. V. S. Sireesha, "Anisotropic universe with cosmic strings and bulk viscosity in a scalartensor theory of gravitation," Astrophysics and Space Science, vol. 335, no. 2, pp. 635-638, 2011.

[18] V. U. M. Rao, K. V. S. Sireesha, and M. V. Santhi, "Bianchi types II, VIII, and IX string cosmological models with bulk viscosity in a theory of gravitation," ISRN Mathematical Physics, vol. 2012, Article ID 341612, 15 pages, 2012.

[19] V. U. M. Rao and K. V. S. Sireesha, "Bianchi types II, VIII and IX string cosmological models with bulk viscosity in BransDicke theory of gravitation," International Journal of Theoretical Physics, vol. 51, no. 10, pp. 3013-3020, 2012.

[20] G. Lyra, "Über eine Modifikation der Riemannschen Geometrie," Mathematische Zeitschrift, vol. 54, pp. 52-64, 1951.

[21] J. Schwinger, Particles, Sources and Fields, Addison-Wesley, Reading, Mass, USA, 1970. 


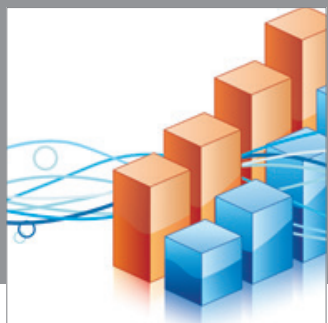

Advances in

Operations Research

mansans

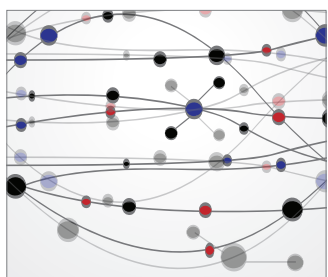

The Scientific World Journal
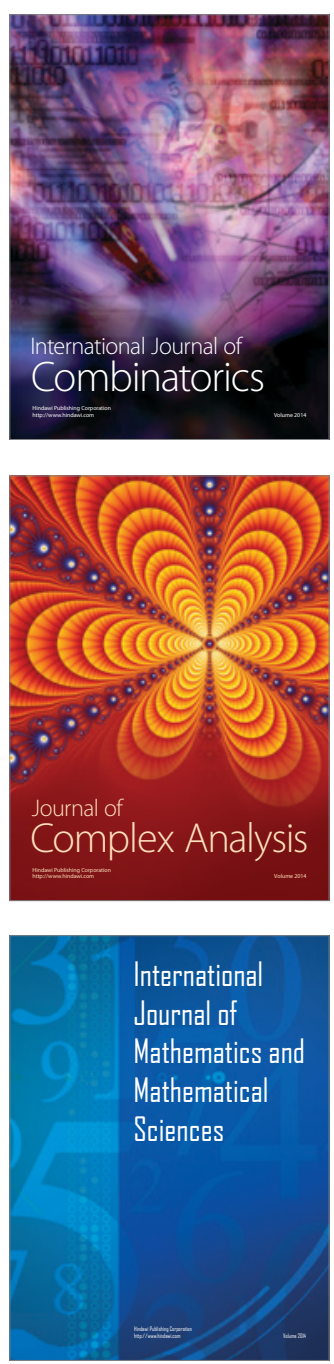
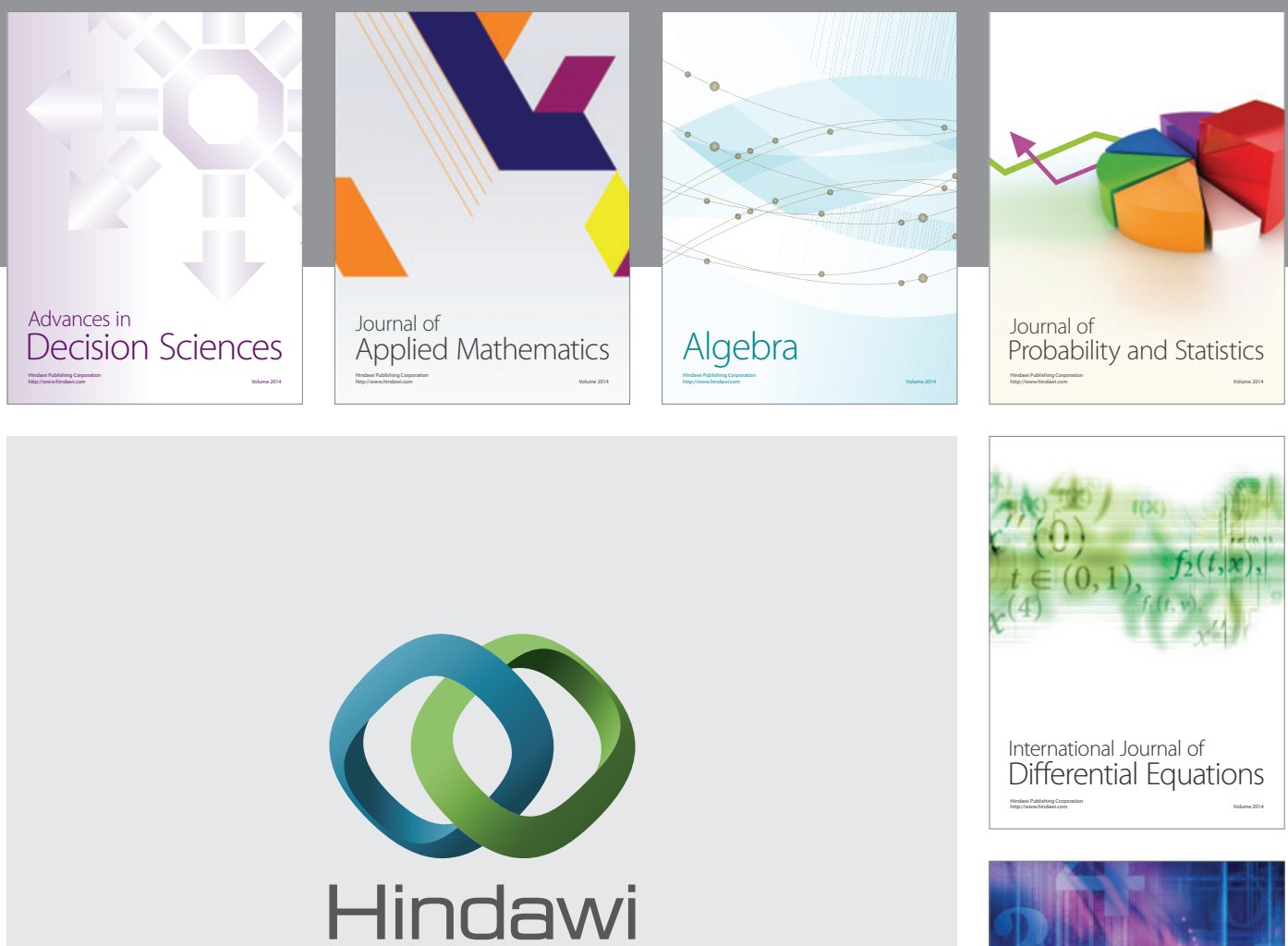

Submit your manuscripts at http://www.hindawi.com
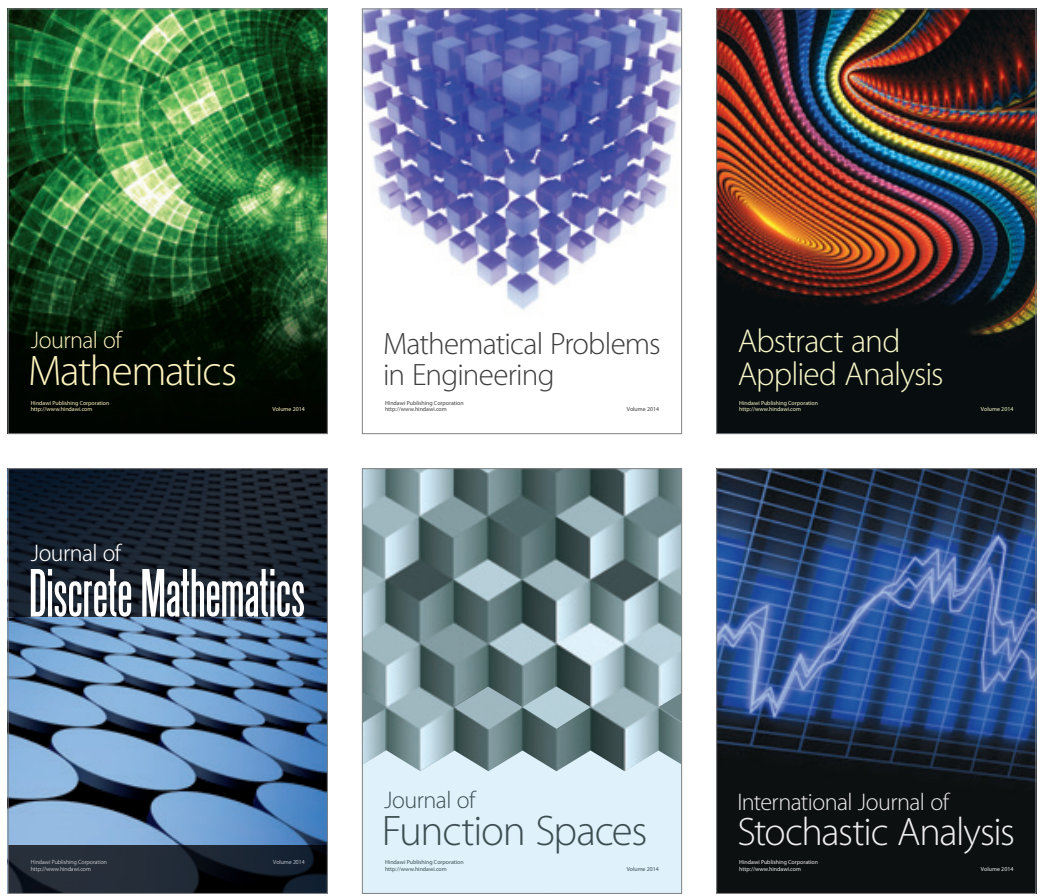

Journal of

Function Spaces

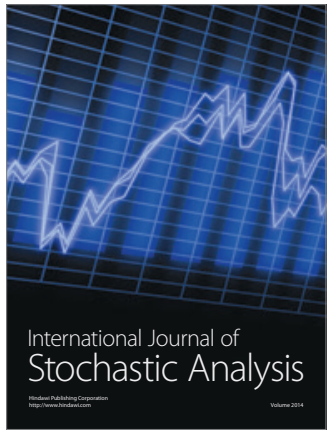

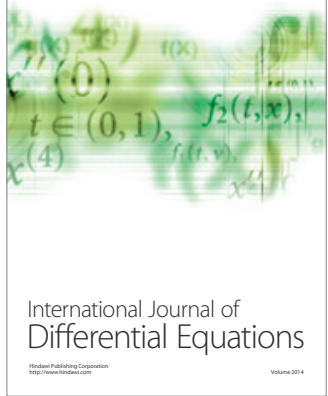
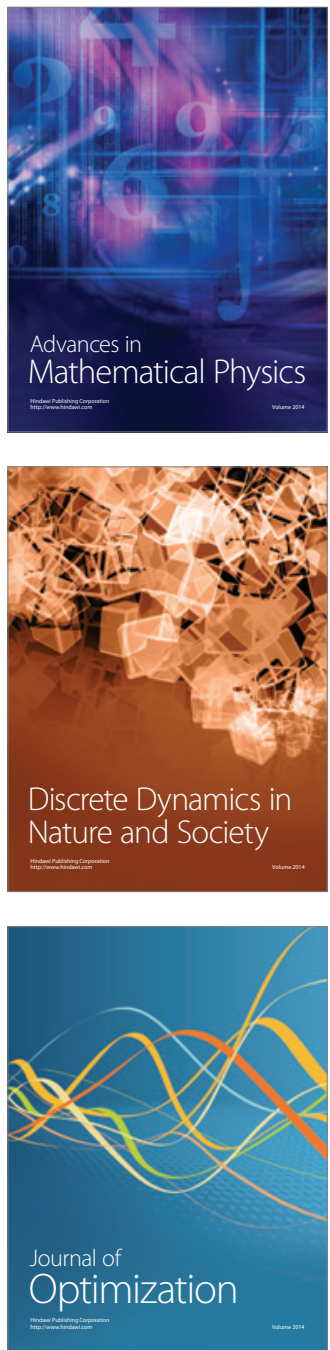